\title{
Assessment of Clinical and Radiological Parameters in Spinal Tuberculosis: Comparison between Human Immunodeficiency Virus-Positive and Human Immunodeficiency Virus-Negative Patients
}

\author{
Shrikant Shrikrishnarao Sagane, Vishal Supda Patil, Girish Dnyaneshwar Bartakke, Kaustubh Yeshwant Kale
}

Department of Orthopaedics, B.J. Govt Medical College and Sassoon General Hospital, Pune, India

\begin{abstract}
Study Design: Prospective comparative study.
purpose: A prospective comparative analysis of 30 patients with spinal tuberculosis (15 human immunodeficiency viruses [HIV] positive and 15 HIV negative). We compared the clinical and radiological parameters of the two groups.

Overview of Literature: With the increasing incidence of HIV and tuberculosis co-infection, spinal tuberculosis is increasing globally, especially in developing countries. The diagnosis of spinal tuberculosis presents a challenge due to nonspecific constitutional symptoms and late presentation.

Methods: A prospective study was conducted of 30 patients with spinal tuberculosis (15 HIV positive and 15 HIV negative) from August 2014-July 2016 for assessment of clinical and radiological parameters. Neurological assessment was done by classification of tuberculous paraplegia, and the amount of kyphosis was assessed by Cobb angle on a plain radiograph. Abscess size in anterior epidural space, the number of vertebral bodies involved and collapsed, and skip lesions were noted on magnetic resonance imaging. Results: In the prospective analysis of 30 patients, HIV positive $(n=15)$ and HIV negative $(n=15)$, there was no significant difference in neurological grading between the two groups. The amount of vertebral body destruction and degree of kyphosis was significantly greater in HIV-negative patients as compared with HIV-positive patients. There was a significant difference in Cobb angle between the two groups. The amount of anterior epidural space abscess formation was greater in HIV-positive patients as compared with HIVnegative patients.

Conclusions: HIV-negative patients had greater vertebral body destruction and resultant kyphosis as compared with HIV-positive patients, who had greater anterior epidural abscess formation.
\end{abstract}

Keywords: Spinal tuberculosis; Human immunodeficiency virus; Kyphosis; Magnetic resonance imaging

\section{Introduction}

Spinal tuberculosis (TB) is the most debilitating form of extrapulmonary TB. The classic destruction of disk space, adjacent vertebral bodies, and involvement of the posterior column of the spine, leading to severe and progressive kyphosis and neurological impairment, were described by Sir Percival Pott in 1779. Thus, it is popularly known as

Received Aug 5, 2019; Revised Sep 5, 2019; Accepted Oct 13, 2019

Corresponding author: Vishal Supda Patil

Department of Orthopaedics, B.J. Govt Medical College and Sassoon General Hospital, Jay Prakash Narayan Road, Pune, Maharashtra 411001, India

Tel: +91-02-27280551, Fax: +91-20-26126868, E-mail: vishalpatil@gmail.com 
Pott's spine [1].

India contributes $23 \%$ of the global TB burden [2]. Skeletal TB accounts for approximately $10 \%$ of extrapulmonary TB and for more than $1 \%-5 \%$ of TB cases worldwide [3]. Spinal TB constitutes more than half of skeletal TB cases. With the pandemic of human immunodeficiency virus (HIV) patients, there is a $27-35$ times greater risk of developing TB. The incidence of skeletal TB infection increases by $60 \%$ in HIV-positive patients $[4,5]$.

Chronic backache is the most common symptom of spinal TB and is found in $61 \%$ of spinal TB patients [6]. The constitutional symptoms of spinal TB include malaise, evening rise of fever, night sweats, generalized body ache, and fatigue. Nonspecific constitutional symptoms and the indolent course of the disease lead to late presentation. This leads to delayed intervention, with increased incidence of neurological impairment and spinal deformity needing correction. Difficulty in establishing early diagnosis, a lack of straightforward guidelines regarding the duration of medical management and need for surgical correction, and the emergence of multidrug-resistant (MDR) strains with the global migration phenomenon pose challenges to the health care system.

Plain radiographs generally remain normal in the early stages of the disease, as $30 \%$ mineral loss is required for the lesions to be appreciated radiographically. Computed tomography scans are useful for the detection of discovertebral lesions and paravertebral abscesses. Magnetic resonance imaging (MRI) remains the gold standard for the early diagnosis of spinal TB. It determines the spread of the disease to the soft tissues and the extent of spinal cord involvement and aids in assessing the response to the treatment. The MRI scan has a reported sensitivity and specificity of $100 \%$ and $88.2 \%$, respectively, for the diagnosis of spinal TB $[7,8]$.

We performed a prospective comparative study of clinical and radiological features of spinal TB in HIV-positive and HIV-negative patients.

\section{Materials and Methods}

\section{Study setting and participants}

We conducted a prospective comparative study in $30 \mathrm{pa}-$ tients with spinal TB visiting our outpatient department (OPD) at the BJ Medical College and Sassoon General Hospital, Pune, from August 2014-July 2016. The study was performed after obtaining approval from the Institutional Ethics Committee (IEC no., d-0615071-71) and written informed consent from all patients. Of these 30 patients with spinal $\mathrm{TB}, 15$ were HIV positive and 15 were HIV negative. All of the patients were assessed with history taking, neurological examination, and radiological examination by X-ray and MRI and CD4 count in HIVpositive cases. Patients presenting with signs and symptoms of spinal TB, involvement of thoracolumbar spinal $\mathrm{TB}$, and aged more than 12 years were included in the study. Patients with cervical spine TB were excluded from the study. The 30 patients were classified into two groups: (1) group 1 (HIV-positive patients) and group 2 (HIVnegative patients).

The history included low backache and constitutional symptoms such as malaise, generalized body-ache, fatigue, evening rise of fever, weight loss, and loss of appetite. Neurological assessment was done by KUMAR Classification of tuberculous paraplegia and tetraplegia based on motor weakness (Table 1) $[9,10]$.

Radiological assessment was done by plain radiograph of the thoracolumbar and lumbosacral spine (anteroposterior and lateral views), and Cobb angle was noted on the plain radiographs.

A 1.5-Tesla GE Healthcare MRI machine (GE Healthcare, Milwaukee, WI, USA) was used for MRIs of the thoracolumbar spine and lumbosacral spine. The following MRI findings were noted in the patients: (1) number of vertebral bodies involved, (2) number of vertebral bodies collapsed, (3) skip lesions, (4) amount of kyphosis, (5) abscess size in anterior epidural space, and (6) pattern of

Table 1. Classification of tuberculous paraplegia and tetraplegia 
vertebral body involvement (paradiscal, anterior, central, and posterior).

\section{Statistical analysis}

The statistical analysis was done with IBM SPSS Software ver. 20.0 (IBM Corp., Armonk, NY, USA). A Pearson chisquare test was used for correlation of age, gender, quantitative vertebral involvement, pattern of vertebral involvement, and degree of kyphosis between the two groups. An independent samples $t$-test was used for abscess size in the anterior epidural space. A $p$-value $<0.05$ was considered statistically significant.

\section{Results}

There was no significant difference between HIV-positive and HIV-negative spinal TB patients with regard to demographic data (age and sex). The mean age of HIV-positive patients was 47 years, and the mean age for HIV-negative patients was 35 years. On applying the chi-square test, $p$ value was 0.32 , which shows that there was no significant difference in the age distribution of patients in the two groups, and hence, the distribution was uniform (Table 2).

In terms of neurological outcome, among HIV-positive patients, $13(86.7 \%)$ had stage 1 neurology and two (13.3\%) had stage 2 neurology. Among HIV-negative patients, 10 (67\%) had stage 1 neurology and five (33\%) had stage 2 neurology. There was no significant difference in neurology between patients in the two groups ( $p=0.195$ ). With regard to the number of vertebrae involved, among HIV-positive patients, two vertebrae were involved in 10 patients (66.7\%), three vertebrae in four patients (26.6\%), and four vertebrae in one patient (6.7\%). Among HIV-

Table 2. Age-wise distribution of cases

\begin{tabular}{lcc} 
Age (yr) & Group 1 (HIV-positive) & Group 2 (HIV-negative) \\
\hline Mean (min-max) & $47(30-68)$ & $35(13-65)$ \\
$10-20$ & 0 & 3 \\
\hline $20-30$ & 0 & 1 \\
$30-40$ & 4 & 5 \\
$40-50$ & 5 & 5 \\
$50-60$ & 3 & 0 \\
$>60$ & 3 & 1 \\
\hline
\end{tabular}

Pearson chi-square value $=22.3 ; p=0.32$ (not significant).

$H I V$, human immunodeficiency virus. negative patients, there was one vertebra involved in one (6.7\%) patient, two vertebrae in 11 patients (73.3\%), and three vertebrae in three patients (20\%). There was no significant difference in the number of vertebrae involved between the two groups $(p=0.53)$. The majority of patients in both groups had paradiscal patterns of vertebral body involvement. In HIV-positive patients, the median Cobb angle was $10^{\circ}$ and the mean was $10.27^{\circ}$. In HIV-negative patients, the median Cobb angle was $20^{\circ}$ and the mean was $17.33^{\circ}$ (Table 3 ). There was a statistically significant difference in the degree of kyphosis in terms of Cobb angle between the two groups ( $p=0.015$ ). Hence, the amount of vertebral body destruction and degree of kyphosis was greater in HIV negative as compared with HIV-positive patients.

The two groups significantly differed in Cobb angle. HIV-negative cases had a larger Cobb angle as compared with HIV-positive cases (Fig. 1). MRI image of HIV negative patient showed significant vertebral body destruction and kyphosis with minimal abscess formation (Fig. 2).

There was no significant difference in skip lesions among patients in the two groups $(p=0.69)$. The mean abscess size in the anterior epidural space was $7.95 \pm 4.34$ $\mathrm{cm}^{2}$ in HIV-positive patients and $1.8 \pm 1.27 \mathrm{~cm}^{2}$ in HIVnegative patients (Table 4 ). Thus, there was a statistically significant difference between the two groups $(p<0.0001)$.

Table 3. Degree of kyphosis measured in terms of $\mathrm{K}$ angle

\begin{tabular}{lcc} 
K angle $\left({ }^{\circ}\right)$ & Group $1($ HIV-positive) & Group 2 (HIV-negative) \\
Median & 10 & 20 \\
Mean & 10.27 & 17.33 \\
\hline
\end{tabular}

Pearson chi-square value $=10.5 ; p=0.015$ (significant). HIV, human immunodeficiency virus.

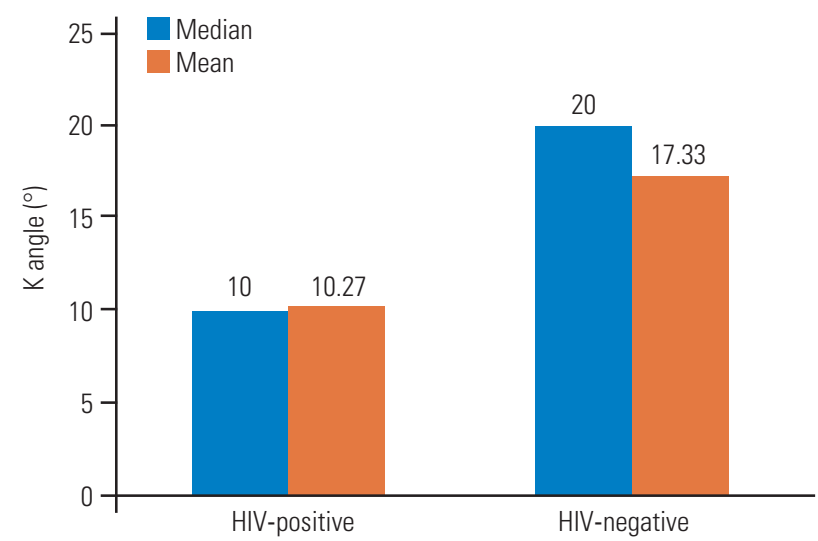

Fig. 1. Figure suggestive of HIV-negative patients having larger $k$ angles as compared with HIV-positive patients. HIV, human immunodeficiency virus. 


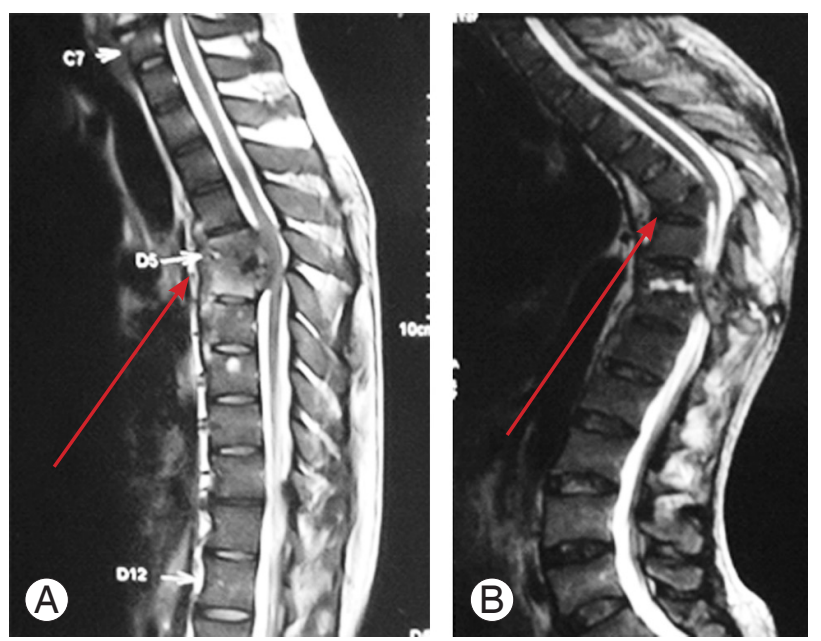

Fig. 2. (A, B) Magnetic resonance imaging image of human immunodeficiency virus-negative patient showing significant vertebral body destruction and kyphosis with minimal abscess formation (arrow).

Table 4. Mean abscess size

$\begin{array}{lcc}\text { Abscess size }\left(\mathrm{cm}^{2}\right) & \begin{array}{c}\text { Group } 1 \\ \text { (HIV-positive) }\end{array} & \begin{array}{c}\text { Group 2 } \\ \text { (HIV-negative) }\end{array} \\ \text { Mean } \pm \text { standard deviation } & 7.95 \pm 4.34 & 1.8 \pm 1.27\end{array}$

Independent samples $t$-test; $F=31.21 ; p<0.0001$ (highly significant) HIV, human immunodeficiency virus.

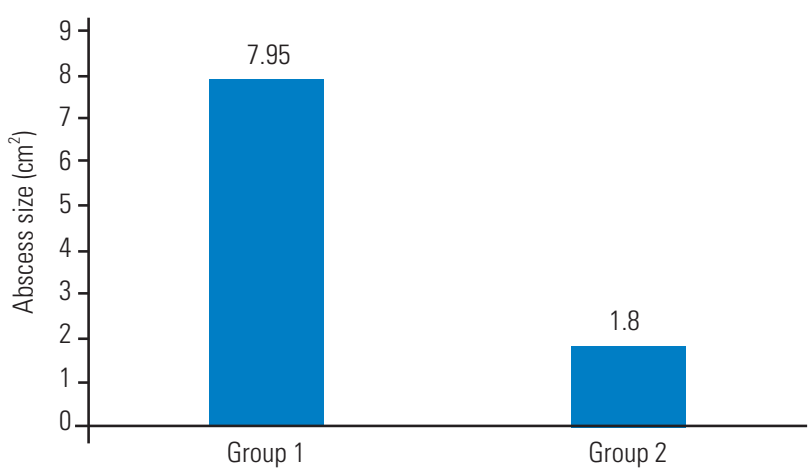

Fig. 3. Abscess size is significantly larger in human immunodeficiency viruspositive patients.

The amount of abscess formation was greater in HIVpositive patients because of decreased cell-mediated immunity and the induction of an inflammatory response by TB infection.

The size of abscess is significantly larger in HIV positive as compared to HIV negative patients (Fig. 3). MRI image of HIV-positive patient showed an excess of abscess formation (Fig. 4).
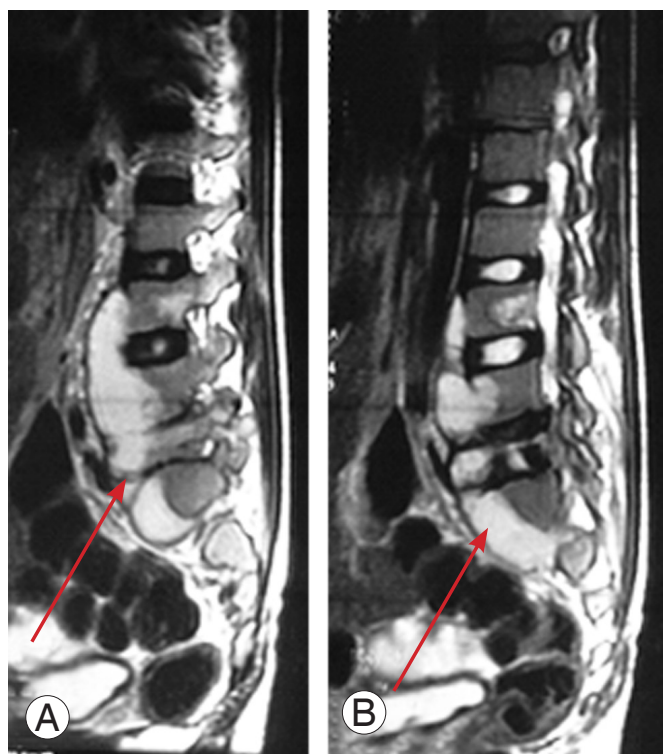

Fig. 4. (A, B) Magnetic resonance imaging of human immunodeficiency viruspositive patient showing an excess of abscess formation (arrow)

\section{Discussion}

Despite effective imaging modalities and management strategies, the increasing prevalence of immunodeficient survivors and the emergence of MDR and extreme drugresistant strains has resulted in a resurgence of all forms of TB worldwide. Delays in presentation and diagnosis are almost universal with spinal TB. Immunosuppressed patients have further delays because of less vigorous inflammation and an impaired immune response. In the absence of standardized guidelines for the management of spinal TB, Tuli's "middle-path" regime is widely accepted and practiced. Multidrug antituberculous therapy (ATT) remains the first line of treatment in both complicated and uncomplicated TB. World Health Organization recommends 9 months of treatment, with an initiation phase of 2 months followed by 7 months of a continuation phase [11]. Surgery is advised only in cases of (1) unresponsiveness to chemotherapy, (2) recurrence, (3) static or worsening neural deficit even after chemotherapy, (4) instability, (5) incapacitating pain, and (6) deformities [12,13]. Early diagnosis and the prompt initiation of ATT remains the key to successful outcomes in spinal TB.

Although a larger concentration of patients affected by spinal TB were males (21, or $70 \%)$, there was no significant difference in the distribution of males and females in the groups of HIV-positive and HIV-negative spinal TB patients. Spinal TB does not have a sexual predilection, 
although studies by Chen et al. [14] and Kandala et al. [15] showed a male preponderance.

In the present study, the mean age of HIV-positive patients with spinal TB was 47 years, and the mean age of HIV-negative patients was 35 years. HIV-positive patients were also older (43 years) in a study by Leibert et al. [16]. These findings were contrary to a study conducted by Anley et al. [17], which showed a younger mean age (32.4 years) of HIV-positive spinal TB patients as compared with the HIV-negative group (45.5 years).

In our study, the majority of patients in the HIV-positive (13, or $86.7 \%)$ and HIV-negative (10, or 67\%) groups had stage 1 neurological grading. HIV-positive patients were referred to our OPD from the antiretroviral therapy center (ART) center because of back pain, and these patients were screened early for spinal TB and diagnosed in the early stages. Hence, there was no neurological involvement observed. A study conducted by Jellis [18] suggested that the clinical presentation of spinal TB in patients infected with HIV is similar to that of patients who are HIV negative; however, the occurrence of spinal TB was more common in persons infected with HIV.

There was no significant difference in the number of vertebral bodies involved in both groups. This is in accordance with a study conducted by Anley et al. [17] in which HIV-positive patients had an average of 3.5 (1-10 2.4$)$ bodies involved as compared with $3.6(2-10 \pm 2.0)$ in HIVnegative patients.

The paradiscal type of vertebral body involvement was observed in 11 (73.3\%) HIV-positive patients and 12 (80\%) HIV-negative patients. Rasouli et al. [19] concluded that the paradiscal pattern, observed in $80 \%$ of cases, was more frequent in spinal TB.

In HIV-positive patients, the median Cobb angle was $10^{\circ}$ and the mean was $10.27^{\circ}$. In HIV-negative patients, the median Cobb angle was $20^{\circ}$ and the mean was $17.33^{\circ}$, and there was a statistically significant difference in the degree of kyphosis between patients in the two groups. The Cobb angle correlates with vertebral body destruction. Thus, in our study, HIV-positive patients had significantly less vertebral body destruction as compared with HIV-negative patients.

The type IV hypersensitivity (delayed type) reaction plays an important role in defense against TB bacilli. $\mathrm{CD} 4+$ cells are decreased in HIV-positive patients, leading to an impaired immune response and a decreased local tissue inflammatory response to the TB bacilli. An

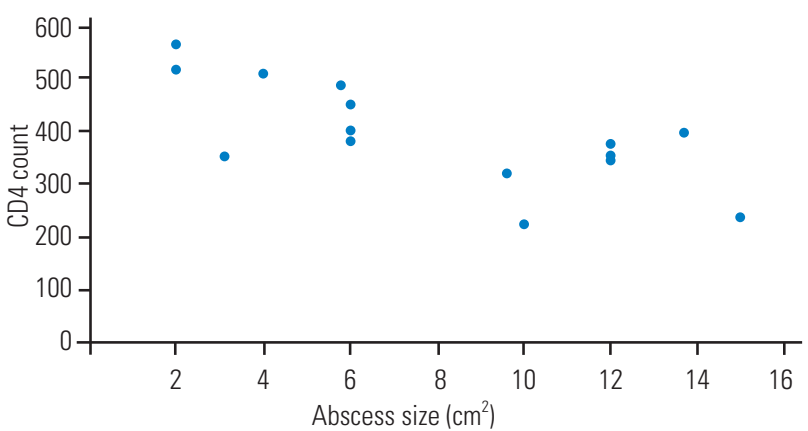

Fig. 5. Correlation of abscess size and CD4 count among human immunodeficiency virus-positive cases.

altered type IV hypersensitivity response in HIV-positive patients makes the typical granulomatous destruction of TB unnoticeable. TB infection in HIV patients also results in an increased secretion of pro-inflammatory mediators, causing increased HIV replication with CD4 cell loss [20]. Fig. 5 shows the correlation between abscess size and CD4 count in HIV-positive patients.

This was in accordance with the study conducted by Anley et al. [17]. The total percentage collapse in HIVnegative patients was significantly higher $(p=0.036)$ at $107 \%(0 \%-235 \% \pm 56.3 \%)$ compared with $75.3 \%$ $(0 \%-235 \% \pm 64.2 \%)$ in HIV-positive patients. The average kyphotic deformity in HIV-positive patients was $9.5^{\circ}\left(0^{\circ}-\right.$ $\left.42^{\circ} \pm 11.8^{\circ}\right)$, whereas the average for HIV-negative patients was $19.2^{\circ}\left(12^{\circ}-40^{\circ} \pm 10.2^{\circ}\right)(p=0.002)$.

The presence of skip lesions in vertebral bodies between the two groups was not statistically significant. Atypical spinal TB includes skip vertebral lesions, isolated spinous process lesions, and neural arch lesions, with skip lesions being the most common form. Skip lesions are reported to be a manifestation of HIV infection, MDR-TB, or chronicity [21].

In our study, the mean abscess size in the anterior epidural space was $7.95 \pm 4.34 \mathrm{~cm}^{2}$ in HIV-positive patients and $1.8 \pm 1.27 \mathrm{~cm}^{2}$ in HIV-negative patients. Hence, the amount of abscess formation was significantly greater in HIV-positive patients because of decreased cell-mediated immunity in patients and the induction of an inflammatory response by $\mathrm{TB}$ infection.

HIV-positive patients show a trend toward a greater epidural abscess volume. This difference may be explained by the reduced autoimmune response of the type IV hypersensitivity reaction caused by the HIV infection. Similar results were found in a study conducted by Anley et al. [17], which suggested a greater epidural abscess volume in 
HIV-positive patients.

As there is increased abscess formation with less vertebral body destruction, simple decompressive surgery like costotransversectomy may be indicated for spinal TB management in HIV-positive patients, whereas decompression and fixation surgeries are required for the correction of deformity in HIV-negative patients because of greater vertebral body destruction.

Some limitations of the study include the small sample size and the fact that HIV-positive patients who had backache were directly referred from the ART center, and hence, correct neurological presentation cannot be judged as these patients were screened early. Further studies may be planned to investigate the correlation between CD4 count and immunity status, the amount of vertebral body involvement, and the neurological status of the patients.

\section{Conclusions}

We conducted this study mainly to assess the differences in clinico-radiological parameters between HIV-positive and HIV-negative groups of spinal TB patients. The HIVpositive patients had larger abscess formation and less vertebral body destruction, leading to lesser kyphotic deformity, and the HIV-negative patients had greater vertebral body destruction with significant kyphotic deformity, leading to early neurological involvement. This effect may be the result of an altered immune response in HIVpositive patients.

\section{Conflict of Interest}

No potential conflict of interest relevant to this article was reported.

\section{ORCID}

Shrikant Shrikrishnarao Sagane: https://orcid.org/0000-00026714-4506; Vishal Supda Patil: https://orcid.org/0000-00029920-469X; Girish Dnyaneshwar Bartakke: https://orcid. org/0000-0002-7635-1398; Kaustubh Yeshwant Kale: https:// orcid.org/0000-0002-8326-2609

\section{References}

1. Kumar K. Tuberculosis of spine: natural history of disease and its judicious management. J Western Pac
Orthop Assoc 1988;25:1-18.

2. Rajasekaran S, Soundararajan DC, Shetty AP, Kanna RM. Spinal tuberculosis: current concepts. Global Spine J 2018;8(4 Suppl):96S-108S.

3. Trecarichi EM, Di Meco E, Mazzotta V, Fantoni M. Tuberculous spondylodiscitis: epidemiology, clinical features, treatment, and outcome. Eur Rev Med Pharmacol Sci 2012;16 Suppl 2:58-72.

4. Garg RK, Somvanshi DS. Spinal tuberculosis: a review. J Spinal Cord Med 2011;34:440-54.

5. Agrawal V, Patgaonkar PR, Nagariya SP. Tuberculosis of spine. J Craniovertebr Junction Spine 2010;1:7485.

6. Cormican L, Hammal R, Messenger J, Milburn HJ. Current difficulties in the diagnosis and management of spinal tuberculosis. Postgrad Med J 2006;82:46-51.

7. Hodgson AR, Wong W, Yau A. X-ray appearance of tuberculosis of the spine. Springfield (IL): Charles C Thomas; 1969.

8. Kim NH, Lee HM, Suh JS. Magnetic resonance imaging for the diagnosis of tuberculous spondylitis. Spine (Phila Pa 1976) 1994;19:2451-5.

9. Tuli SM. Judicious management of tuberculosis of bone joint and spine. Indian J Orthop 1985;19:14766.

10. Tuli SM. Tuberculosis of the spine: a historical review. Clin Orthop Relat Res 2007;460:29-38.

11. World Health Organization. Treatment of tuberculosis: guidelines for treatment of drug-susceptible tuberculosis and patient care: 2017 update. Geneva: World Health Organization; 2017.

12. Tuli SM. Results of treatment of spinal tuberculosis by "middle-path" regime. J Bone Joint Surg Br 1975;57:13-23.

13. Shetty A, Kanna RM, Rajasekaran S. TB spine: current aspects on clinical presentation, diagnosis, and management options. Semin Spine Surg 2016;28:15062.

14. Chen CH, Chen YM, Lee CW, Chang YJ, Cheng CY, Hung JK. Early diagnosis of spinal tuberculosis. J Formos Med Assoc 2016;115:825-36.

15. Kandala M, Sugnaneswar P, Vaishnavi V, et al. Clinico radiological correlation with histopathological and molecular diagnosis in spinal tuberculosis. MedPulse Int J Orthop 2017;4:1-5.

16. Leibert E, Schluger NW, Bonk S, Rom WN. Spinal tuberculosis in patients with human immunodefi- 
ciency virus infection: clinical presentation, therapy and outcome. Tuber Lung Dis 1996;77:329-34.

17. Anley CM, Brandt AD, Dunn R. Magnetic resonance imaging findings in spinal tuberculosis: comparison of HIV positive and negative patients. Indian J Orthop 2012;46:186-90.

18. Jellis JE. Human immunodeficiency virus and osteoarticular tuberculosis. Clin Orthop Relat Res 2002;(398):27-31.

19. Rasouli MR, Mirkoohi M, Vaccaro AR, Yarandi KK,
Rahimi-Movaghar V. Spinal tuberculosis: diagnosis and management. Asian Spine J 2012;6:294-308.

20. Danaviah S, Sacks JA, Kumar KP, et al. Immunohistological characterization of spinal TB granulomas from HIV-negative and -positive patients. Tuberculosis (Edinb) 2013;93:432-41.

21. Wu M, Su J, Yan F, Cai L, Deng Z. Skipped multifocal extensive spinal tuberculosis involving the whole spine: a case report and literature review. Medicine (Baltimore) 2018;97:e9692. 